Aus der Phoniatrischen und Pädaudiologischen Abteilung der Hals-Nasen-Ohren-Klinik der

Friedrich-Alexander-Universität Erlangen-Nürnberg

Leiter: Prof. Dr. med. Dr. rer. nat. U. Eysholdt

\title{
Sprachverständlichkeit von Kindern mit bilateralen und unilateralen Lippen-Kiefer-Gaumen-Spalten
}

\author{
Inaugural-Dissertation \\ zur Erlangung der Doktorwürde \\ der Medizinischen Fakultät \\ der \\ Friedrich-Alexander-Universität \\ Erlangen-Nürnberg
}

vorgelegt von

Franziska Dames

aus

Meerane 


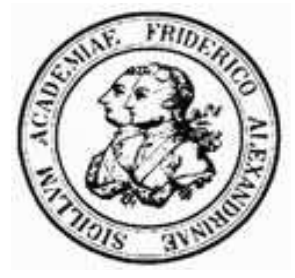

Gedruckt mit Erlaubnis der

Medizinischen Fakultät der Friedrich-Alexander-Universität Erlangen-Nürnberg

Dekan:

Referent:

Korreferent:
Prof. Dr. J. Schüttler

Prof. Dr. Dr. U. Eysholdt

Priv.-Doz. Dr. M. Schuster

Tag der mündlichen Prüfung: 25. November 2009 


\section{Inhaltsverzeichnis}

Seitenzahl

\section{Zusammenfassung}

1.1 Hintergrund und Ziele

1.2 Methode

1.3 Ergebnisse

1.4 Schlußfolgerung

2. Abstarct

2.1 Objective

2.2 Methods

2.3 Results

2.4 Conclusions

3. Einleitung

4. Patienten und Methode

4.1 Patienten

4.2 Kontrollgruppe

4.3 Testmaterial

4.4 Spracherkennungssystem

4.5 Analyse und Auswertung der Daten

\section{Ergebnisse}

6. Diskussion

6.1 Fazit

7. Tabellen

7.1 Tabelle 1: Patienten und Kontrollgruppe

7.2 Tabelle 2: Ergebnisse der Sprachverständlichkeitsprüfung (Worterkennungsrate WR)

7.3 Tabelle 3: Vergleich zwischen weiblichen und männlichen Probanden

8. Abbildungen

8.1 Abbildung 1

8.2 Abbildung 2

9. Literaturverzeichnis

10. Danksagung 


\section{Zusammenfassung}

\subsection{Hintergrund und Ziele}

Zur Auswirkung einer einseitigen oder beidseitigen Lippen-KieferGaumenspaltfehlbildung (LKG) auf die Sprachqualität liegen in der Literatur unterschiedliche Ergebnisse vor. In dieser Studie wurde objektiv mittels automatischer Spracherkennungstechnik die Verständlichkeit von Kindern mit unilateraler und bilateraler LKG ermittelt.

\subsection{Methode}

Bei 72 deutschsprachigen Kindern (8,7 $\pm 3,0$ Jahre) mit LKG, davon 17 mit bilateraler, 23 mit rechtsseitiger und 32 mit linksseitiger LKG, wurde der Verständlichkeitsgrad als prozentualer Anteil korrekt erkannter Wörter eines standardisierten Sprachentwicklungstests objektiv ermittelt (Worterkennungsrate WR) und mit einer Kontrollgruppe aus 159 Kindern $(9,1 \pm 2,9$ Jahre) verglichen.

\subsection{Ergebnisse}

Zwischen den Spaltgruppen LKG rechts, links und beidseits zeigte sich kein signifikanter Unterschied. Zur Kontrollgruppe (WR Mittelwert 63,5\% $\pm 12,1 \%$ ) weist das Patientenkollektiv (WR Mittelwert 48,1\% \pm 16,3\%) einen signifikanten Unterschied auf $(p<0,001)$. Die WR steigt mit zunehmendem Alter bei der Kontrollgruppe und den Patientengruppen mit unilateraler Spaltfehlbildung signifikant an, was bei den Kindern mit bilateraler LKG bei breiter Streuung der WR nicht festzustellen ist. In dieser Gruppe liegt die WR bei den Jungen auch leicht signifikant höher als bei den Mädchen. In der Kontrollgruppe so wie in den Patientengruppen mit unilateraler Spaltfehlbildung findet sich bezüglich der WR kein signifikanter Unterschied zwischen den Geschlechtern.

\subsection{Schlußfolgerung}

Trotz der größeren Ausdehnung der Fehlbildung bei Kindern mit bilateralen LKG zeigt sich keine signifikante Abweichung des Verständlichkeitsgrades zu den Kindern mit einseitiger LKG. 


\section{Abstract}

\subsection{Objective}

Data about the effect of unilateral or bilateral cleft lip and palate (CLP) on speech quality are inconsistent. In this study we firstly quantify the intelligibility of children with unilateral and bilateral CLP objectively by means of automatic speech recognition system (ASR).

\subsection{Methods}

Speech data of 72 German speaking children (8.7 \pm 3.0 years) with CLP thereof 17 children with bilateral CLP, 23 with CLP on the right side, and 32 on the left, were compared. A group of 159 children aged $9.1 \pm 2.9$ years served as control group. To quantify intelligibility we calculated the word recognition rate (WR) as the percentage of correctly recognized words of a standardized speech test (PLAKSS).

\subsection{Results}

Between the 3 cleft groups, there was no significant difference in WR. Compared to the control group (WR mean 63.5\% $\pm 12.1 \%$ ), the patient group (WR mean $48.1 \% \pm$ $16.3 \%)$ shows significant lower WR $(p<0.001)$. The WR rises with increasing age in the control group and in the patient groups with unilateral cleft significantly. This couldn't be observed in the children with bilateral CLP. In this group the males showed a significantly higher WR than the females. In the control group as in the patient groups with unilateral cleft there is no significant difference between girls and boys.

\subsection{Conclusions}

Despite the greater extent of the malformation of children with a bilateral CLP, there is no significant difference of WR to the children with unilateral cleft lip and palate. 


\section{Einleitung}

Lippen-Kiefer-Gaumen-Spaltfehlbildungen (LKG) zählen zu den häufigsten Fehlbildungen im Kopfbereich [27]. Sie beeinflussen die Ästhetik der Patienten sowie die Nahrungsaufnahme, die Mimik, die Mittelohrbelüftung und das Sprechen [7, 19, 20, 21] und haben damit Auswirkungen auf die psychosoziale Kompetenz [2, 5]. Typische Sprechstörungen von Patienten mit LKG sind der nasale Durchschlag und Hyperrhinophonie, abgeschwächte Artikulationsspannung und die Verlagerung der Artikulation (Rück- oder Vorverlagerung) [6, 33]. Nasaler Durchschlag, Hyperrhinophonie sowie abgeschwächte Sprechspannung sind das Produkt struktureller Abnormalitäten oder Dysfunktionen und werden als passive Änderungen bezeichnet. Passive Änderungen können im Gegensatz zu aktiven (Verlagerung der Artikulation) durch chirurgische Therapie verbessert werden [6]. Übende Verfahren wie die Optimierung des gesamtkörperlichen und Velumtonus, phonetisch-phonologische Therapie oder Biofeedback mittels flexibler Nasopharyngoskopie [1] werden für beides eingesetzt.

In der Literatur wird bislang beschrieben, dass bilaterale LKG im Vergleich zu unilateralen LKG einen bedeutend höheren Einfluss auf die Verständlichkeit haben. Durch größere anatomische Deformationen bei bilateralen LKG entstehen stärkere Sprechprobleme, welche verglichen mit unilateralen LKG zusätzlich ein größeres Maß an logopädischer Therapie erfordern [3, 8, 10, 25]. Lohmander et al. fanden in ihrer Studie 1995 ebenso wie Van Lierde et al. (2002 sowie 2003) jedoch keinen signifikanten Unterschied zwischen den Spalttypen (LKG unilateral und bilateral) und Sprachergebnissen [13, 28, 29].

Um den Verständlichkeitsgrad diagnostizieren zu können, bewerteten erfahrene oder naive Hörer Patienten mit Spaltfehlbildungen bislang perzeptiv, während Stimmqualität oder nasale Luftströmungen bei der Phonation durch schon längere Zeit zur Verfügung stehende objektive Verfahren gemessen wurden. Allerdings ist bekannt, dass die auditive Bewertung teils bedingt durch unterschiedliche Erfahrung der Hörer, eine bedeutsame Inter-Bewerter-Varianz aufweisen kann [9]. Dadurch kann die Aussagekraft von klinischen Studien eingeschränkt werden.

Zur Verständlichkeitsbewertung findet sich erst seit kurzem eine geeignete objektive Methode, welche auf der Technologie von Spracherkennungssystemen basiert. Diese nutzen die phonematischen Eigenschaften der Sprache, um unbekannte Wörter oder Wortfolgen zu erkennen. Um die Verständlichkeit von Patienten bewerten zu können, wird das Verfahren umgekehrt genutzt - bei bekannten Wörtern kann entsprechend der Erkennungsrate auf die Qualität des Gesprochenen geschlossen werden. Das 
System ist so in der Lage, auf die Verständlichkeit eines Patienten beim Lesen oder Nachsprechen eines Textes rückzuschließen. Durch die automatische Bewertung können Wortakkuratheit (WA) und Worterkennungsrate (WR) als prozentual richtig erkannte Wörter einer Wortreihe berechnet und folglich der Verständlichkeitsgrad ermittelt werden.

In einer Pilotstudie von 2006 wurde zwischen der subjektiven Bewertung der Verständlichkeit von Kindern mit Spaltfehlbildungen durch mehrere Experten und der objektiven Methode der ASR eine hohe Übereinstimmung nachgewiesen $(r=-0,9, p<$ 0,01 ) [23]. Eine objektive Evaluation der Verständlichkeit mittels automatischer Spracherkennungstechnik von Schulkindern mit isolierter und kombinierter Gaumenspalte stammt aus der selben Arbeitsgruppe. Hierbei wurde der Verständlichkeitsgrad von Kindern mit Gaumenspalte (GS) mit dem von Kindern mit LKG verglichen. Beide Gruppen wurden bezüglich des Verständlichkeitsgrades jeweils einer altersentsprechenden Kontrollgruppe gegenübergestellt. Es ergaben sich keine signifikanten Unterschiede des Verständlichkeitsgrades zwischen Kindern mit GS und solchen mit LKG, jedoch war bei beiden der Verständlichkeitsgrad (42\% $\pm 17 \%)$ signifikant niedriger als bei der Kontrollgruppe (62\% $\pm 10 \%)$ [31]. Die Ausprägung der LKG Spalten wurde in dieser Studie jedoch nicht berücksichtigt.

In dieser Studie wird nun erstmals ein objektiver Vergleich der Verständlichkeit zwischen Mädchen und Jungen mit verschieden Formen von LKG (bilateral, unilateral links und unilateral rechts), die bislang keine sprechverbessernde Operation erhielten, aufgezeigt und einer altersangepassten Kontrollgruppe gegenübergestellt. 


\section{Patienten und Methode}

\subsection{Patienten:}

Die Probanden für diese Studie wurden aus dem Patientenkollektiv der interdisziplinären Spaltsprechstunde am Spaltenzentrum der Universität ErlangenNürnberg im Zeitraum von Januar 2006 bis einschließlich Januar 2008 ausgewählt. Mit Einverständnis der Eltern wurden von 72 Kindern im Alter von 3,9 bis 14,6 Jahren (Mittelwert 8,7 \pm 3,0 Jahre) digitalisierte Sprachaufnahmen erstellt. Hiervon wiesen 17 Kinder eine bilaterale Lippen-Kiefer-Gaumen-Spalte, 23 eine rechtsseitige unilaterale und 32 Kinder ein linksseitige unilaterale LKG auf (Tabelle 1). Alle 72 Kinder sind deutschsprachig aufgewachsen, einige sprachen einen lokalen Dialekt. Die chirurgische Versorgung der Fehlbildung jedes Kindes erfolgte entsprechend dem Vorgehen des Erlanger Spaltenzentrums. Im Alter von 3 bis 6 Monaten erfolgte der Spaltverschluss von Lippe und Kiefer mit Nasenbodenbildung und Aufrichtung der Nasenspitze in der Technik nach Tennison-Randall. Mit 10 bis 11 Monaten erfolgt der Verschluss des harten und weichen Gaumens nach Langenbeck und Widmaier. Sekundäre osteoplastische Eingriffe (z.B. Kieferspaltosteoplastik) werden bei Bedarf zwischen dem 8. und 12. Lebensjahr durchgeführt. Bei keinem der Kinder wurde vor den Sprachaufnahmen eine sprechverbessernde Operation durchgeführt. Das Kollektiv enthielt nur Kinder, deren Sprachfähigkeit nicht durch weitere funktionelle, neurologische oder psychische Alterationen, außer der Spaltfehlbildung, eingeschränkt war.

\subsection{Kontrollgruppe:}

Es wurden von 159 deutschsprachigen Kindern ohne Spaltfehlbildung einer Erlanger Grundschule sowie eines Erlanger Kindergartens digitalisierte Sprachaufnahmen erstellt. Das Vorgehen entsprach den Sprachaufnahmen bei Spaltkindern. Der lokale Dialekt der Kinder entsprach dem der Patientengruppe. Entwicklungsbedingte Abweichungen der Lautbildung, z.B. durch den Zahnwechsel, wurden als physiologisch angesehen und nicht im Sinne eines Ausschlusskriteriums berücksichtigt. Sprachvermögen und Sprachentwicklung der Kinder der Kontrollgruppe wurde als physiologisch eingeschätzt. Die Kinder der Kontrollgruppe befanden sich im Alter von 4,2 bis 14,9 Jahren (Mittelwert 9,1 \pm 2,9 Jahre).

\subsection{Testmaterial:}

Der aus 99 Wörtern auf 33 Bildtafeln bestehende PLAKSS (Psycholinguistische Analyse kindlicher Sprechstörungen) wurde als Grundlage für die Sprachaufnahmen 
verwendet. Die Testbilder sind dem Wortschatz der Kinder angepasst und sind so ausgewählt, dass alle Laute und die wesentlichen Lautverbindungen der deutschen Sprache in allen Wortpositionen untersucht werden können [4]. Die Sprachaufzeichnung erfolgte bei allen Kindern digital mit einem Call $4 \mathrm{U}$ ComfortHeadset (dnt, Dietzenbach, Deutschland, 16 kHz/ 16 bit).

\subsection{Spracherkennungssystem:}

Um den Verständlichkeitsgrad von Patienten mit Sprachproblemen objektiv bestimmen zu können, wurde am Lehrstuhl für Mustererkennung der Friedrich-AlexanderUniversität Erlangen/Nürnberg ein automatisches Spracherkennungssystem entwickelt, welches erfolgreich validiert und im Rahmen wissenschaftlicher Studien eingesetzt wurde [16, 22, 23, 31]. Zur Nutzung des Spracherkennungssystems wurde das Programm zur Evaluation und Analyse kindlicher Sprachstörungen (PEAKS) eingesetzt [14]. Mit PEAKS besteht die Möglichkeit der Durchführung und Aufnahme verschiedener Tests (z.B. PLAKSS-Test, Lesetexte wie „Nordwind und Sonne“) zur vollautomatischen Bewertung der Verständlichkeit. In dieser Untersuchung wird als $\mathrm{Maß}$ für die Verständlichkeit die Worterkennungsrate, also der Prozentsatz korrekt erkannter Wörter einer Wortreihe, genutzt. Das System wurde so angepasst, dass syntaktische Grundlagen der Erkennung keine Rolle spielen.

Die Durchführung der Verständlichkeitstests dieser Studie erfolgte standardisiert mit PEAKS sowohl bei den Patienten der interdisziplinären Spaltsprechstunde als auch bei den Kindern der Kontrollgruppe.

\subsection{Analyse und Auswertung der Daten}

Für die statistische Auswertung fand das Programm SPSS 16.0 Anwendung. Die Hypothese der Normalverteilung wurde mit dem Kolmogorow-Smirnow-Test überprüft. Es wurde bezüglich der einzelnen Spalttypen sowie der Kontrollgruppe zur Worterkennungsrate der Levene-Test eingesetzt, um die verschiedenen Varianzen auf ihre Homogenität zu testen. Zusätzlich sind der Welch-Test, A-priori-Kontraste und Post-Hoc-Tests verwendet wurden. Die Ermittlung der Ergebnisse in Hinsicht auf die Faktoren Alter und Geschlecht erfolgte durch SPSS mittels t-Tests und der bivariaten Korrelation nach Pearson. 


\section{Ergebnisse}

Im gesamten Patientenkollektiv (LKG rechts, links und beidseits) variiert die Worterkennungsrate WR zwischen 9,1\% und 78,8\% (Mittelwert: 48,1\% $\pm 16,3 \%$ ) und zeigt einen signifikanten Unterschied $(p<0,001)$ zu der Kontrollgruppe. Bei dieser lag die WR zwischen $31,3 \%$ und $85,9 \%(63,5 \% \pm 12,1 \%)$. Beim Vergleich der einzelnen Spalttypen bezüglich der Worterkennungsrate zeichnet sich kein signifikanter Unterschied ab. Die Streuung der Werte ist für die Kinder mit LKG deutlich größer als bei der Kontrollgruppe (Abbildung 1, Tabelle 2).

Die Worterkennungsrate WR steigt mit zunehmendem Alter bei der Kontrollgruppe sowie bei den 2 Patientengruppen mit je unilateralen LKG signifikant an wie in Abbildung 2 dargestellt ( $p<0,001$ ). Bei der Patientengruppe mit bilateraler LKG zeigt sich allerdings kein signifikanter Anstieg der WR bei zunehmendem Alter der Kinder $(p=0,060)$ (Abbildung 2).

Beim Vergleich der Worterkennungsrate der Geschlechter innerhalb der Testgruppen ergab der t-Test keinen signifikanten Unterschied zwischen männlichen und weiblichen Kindern in der Kontrollgruppe $(p=0,701)$, in der Patientengruppen mit rechtsseitiger LKG ( $p=0,581)$, sowie bei den Kindern mit linksseitiger LKG $(p=0,08)$. In der Gruppe der Kinder mit beidseitiger Lippen-Kiefer-Gaumen-Spalte fand sich bei den Jungen ein signifikant besseres Ergebnis der WR als bei den Mädchen ( $p=0,034)$. (Tabelle 3)

\section{$\underline{\text { 6. Diskussion }}$}

In dieser Studie wird zum Vergleich der Verständlichkeit von Kindern mit einseitiger und beidseitiger Lippen-Kiefer-Gaumen-Spalte eine neue computerbasierte Methode angewandt. In vielen vorangegangenen Studien fand die Messung des Verständlichkeitsgrades perzeptiv und somit subjektiv statt $[8,11,13,14,24,25,26$, 27, 32]. Untersuchungen von Keuning et al. 1999 sowie Paal et al. 2005 ergaben, dass durch uneinheitliche Erfahrungen der Hörer und unterschiedliche Vorraussetzungen die subjektiven Bewertungen der Verständlichkeit nur als bedingt verlässlich angesehen werden können [9, 19]. Da durch das computerbasierte System der Verständlichkeitsgrad untersucherunabhängig ermittelt wird, kann die Methode als objektiv gelten und weist eine hohe Reliabilität auf. Durch eine standardisierte Durchführung der Berechnung ist eine gute Vergleichbarkeit gegeben. Ebenso hat sich 
das automatische Spracherkennungssystem durch seine einfache internetbasierte und damit plattformunabhängige Anwendung als eine zeitsparende Methode bewährt, welche dadurch auch keine wesentliche Mehrbelastung für die zu untersuchenden Kinder darstellt.

Analog zu unserem Patientenkollektiv finden sich in der Literatur bezüglich der Angaben zur Inzidenz mehr Kinder mit unilateraler als mit bilateraler Lippen-KieferGaumen-Spalte [8, 10, 13, 18] (siehe Tabelle 1). Im Vergleich zu den genannten Studien sind die Gruppengrößen ausreichend, um eine angemessene statistische Aussagekraft zu erzielen. Eine systematische Verfälschung wurde durch das entsprechende Alter und den entsprechenden Dialekt der Kontrollgruppe verhindert.

Der Verständlichkeitsgrad des Vergleichskollektivs ist, wie hypothetisch angenommen, besser als der Verständlichkeitsgrad der LKG Patienten. Dies entspricht den Aussagen der anderen hier genannten Studien. Außerdem ist erwartungsgemäß innerhalb der Kontrollgruppe ein signifikanter Anstieg der Worterkennungsrate mit steigendem Alter der Grundschul- und Kindergartenkinder zu verzeichnen. Dies ist durch die Spracherkennungstechnologie bedingt, die v. a. mit erwachsenen Sprechern trainiert wurde. Im Patientenkollektiv findet sich nur in den Gruppen mit den Kindern mit unilateraler LKG ein signifikanter Anstieg der Worterkennungsrate mit dem Alter. Die Patientengruppe mit beidseitiger Fehlbildung stellt die kleinste Gruppe in unserer Studie dar. Bei starker Streuung der Daten (Tabelle 2) lässt sich kein signifikanter Anstieg der Worterkennungsrate mit zunehmendem Alter nachweisen (Abbildung 2). Es zeigt sich kein signifikanter Unterschied der Worterkennungsraten zwischen den Patientengruppen mit einseitiger und beidseitiger LKG. Die Literatur stimmt teilweise mit diesem Ergebnis überein [13, 23, 28, 29]. Aber es finden sich auch Studien, die einen signifikanten Unterschied der Verständlichkeit zwischen Kindern mit unilateraler Lippen-Kiefer-Gaumen-Spalte und denen mit bilateraler LKG nachweisen [3, 8, 10, 18, 25]. Hierbei wurden die Kinder mit bilateraler LKG als schlechter eingestuft. Dalston et al. stellten fest, dass die beidseitig belasteten Kinder vermehrte Lautfehlbildung durch kompensatorische Artikulation aufwiesen und somit negative Auswirkung auf die Verständlichkeit zu verzeichnen waren [3]. Neben der signifikant schlechteren Verständlichkeit diagnostizierte man bei den Kindern mit bilateraler LKG in der Studie von Nakajima 2001 zusätzlich, dass diese später Zwei-Wortäußerungen nutzten, also insgesamt eine Sprachentwicklungsverzögerung im Verhältnis zu Kindern mit einseitiger Spaltfehlbildung aufwiesen [18]. Ein Zusammenhang zur Lautbildung oder zur Verständlichkeit ist damit aber noch nicht hinreichend erklärbar. Außerdem stellte Karling 1993 fest, dass Kinder mit beidseitiger LKG mehr logopädische Therapie 
benötigten [8]. Die Studie von Schuster et al. von 2006, bei welcher ebenso wie in unserer Studie eine objektive Meßmethode zum Einsatz gekommen ist, bekräftigt wiederum das vorliegende Ergebnis [23].

Von Van Lierde et al. 2002 wurde die Sprache der Spaltträger subjektiv von 3 Logopäden anhand einer 4 Punkte Skala bewertet und es konnte ebenso kein signifikanter Unterschied zwischen den Patienten mit bilateralen und unilateralen Spaltfehlbildung aufgezeigt werden [29].

Aus dem Vergleich der Verständlichkeit bzw. des Verständlichkeitsgrades von Kindern mit LKG und isolierter Gaumenspalte ist bekannt, dass eine Fehlbildung im Bereich der vorderen Artikulationszone wenig bedeutsam für die Verständlichkeit der Kinder zu sein scheint, da auch hier kein signifikanter Unterschied gemessen mit der automatischen Spracherkennung festgestellt werden konnte [26, 31]. Der Zusammenhang zwischen Lautfehlbildungen der vorderen Artikulationszone und der Verständlichkeit wird in Zukunft in weiteren Studien darlegt.

Die recht unterschiedlichen Vorgehensweisen der verschiedenen Studien belegen die Notwendigkeit einer präzisen und genauen Bewertung der Sprache. Lohmander et al 2004 und die britische Arbeitsgruppe um Sell forderten in ihren Arbeiten standardisierte Bewertungsprozeduren für die Sprache, um Studien besser untereinander vergleichen zu können [12, 24]. Schon Wyatt 1996 meinte, dass die Verständlichkeit schwer zu bewerten sei, weil diese von vielen Variablen wie zum Beispiel der Sprechgeschwindigkeit, der „Scharfsinnigkeit“ des Hörers oder auch von Hintergrundgeräuschen beeinflusst wird [34]. Mit der objektiven computerbasierten Methode ist nun die Möglichkeit einer besseren Vergleichbarkeit gegeben.

Auch bezüglich der Geschlechtsabhängigkeit des Verständlichkeitsgrades von Kindern mit einseitiger oder beidseitiger LKG finden sich in der Literatur kaum Ergebnisse. In unserer Studie besteht zwischen den Geschlechtern kein Unterschied außer in der Gruppe der Kinder mit bilateraler LKG. Die Jungen mit bilateraler LKG wiesen eine signifikant höhere WR auf als die Mädchen (siehe Tabelle 3). Erstrebenswert wäre dieses Ergebnis durch weitere Studien an einem größeren Patientenkollektiv unterstreichen zu können, denn eine Erklärung für das vorliegende Ergebnis mag die eher kleine Gruppengröße der Kinder mit bilateraler LKG sein.

Die Notwendigkeit regelmäßiger Kontrollen der Sprachentwicklung von Kindern mit Spaltfehlbildungen wird durch die aktuellen Ergebnisse unterstrichen, um den individuellen Therapiebedarf bestimmen zu können. Der Einsatz der automatischen Spracherkennung bietet weiterhin die Möglichkeit, die Effektivität des klinischen 
Vorgehens bzw. unterschiedliche Therapiestrategien mit einer einfachen und schnellen Methode in klinischen Studien zu prüfen. Natürlich müssen in einer detaillierten Bewertung der Sprachentwicklung eines Kindes mit Spaltfehlbildung das Ausmaß und die Art einer Lautfehlbildung bestimmt werden. Dies ist bislang perzeptiv erfolgt und ist - je nach Ausmaß - sehr zeitintensiv und benötigt reichlich Erfahrung des Bewerters. Auch hierzu wäre also eine automatisierte Bewertungsmethode hilfreich. Vorarbeiten für eine schnelle und detaillierte Ermittlung phonetischer Abweichungen mittels automatischer Spracherkennung sind daher bereits erfolgt und sind viel versprechend [15]. Diese Anwendung wird ebenfalls bald möglich sein und damit die automatische Bewertung der Verständlichkeit durch die automatische Bewertung der Lautbildungsstörungen ergänzen. Darüber hinaus ist eine Anwendung in anderen Sprachen möglich und wird bereits von einer belgischen Arbeitsgruppe für andere Anwendungen eingesetzt [17, 30].

\subsection{Fazit}

Kinder mit einer doppelseitigen Lippen-Kiefer-Gaumen-Spalte weisen keine geringere Worterkennungsrate als Maß für die Verständlichkeit auf als solche mit einseitiger Lippen-Kiefer-Gaumen-Spalte. Die kontroversen Ergebnisse in der Literatur verlangen nach einer einheitlichen Prozedur, um die klinischen Ergebnisse besser miteinander vergleichen zu können. Die automatische Spracherkennung bietet ein objektives und wenig aufwändiges Verfahren, um die Verständlichkeit als übergeordneten Parameter für die Qualität des Sprechens zu beurteilen. 


\section{Tabellen}

7.1 Tabelle 1: Patienten und Kontrollgruppe

\begin{tabular}{|l|c|c|c|c|}
\hline & Anzahl & $\begin{array}{c}\text { Alter (Mittelwert, } \\
\text { Standardabweichung) }\end{array}$ & Alter min & Alter max \\
\hline \hline Kontrollgruppe & 159 & $9,1 \pm 2,9$ & 4,2 & 14,9 \\
\hline LKG unilateral rechts & 23 & $8,8 \pm 2,7$ & 3,9 & 13,4 \\
\hline LKG unilateral links & 32 & $8,7 \pm 3,3$ & 4 & 14,6 \\
\hline LKG bilateral & 17 & $8,8 \pm 3,1$ & 4,1 & 13,6 \\
\hline
\end{tabular}

\subsection{Tabelle 2: Ergebnisse der Sprachverständlichkeitsprüfung} (Worterkennungsrate WR)

\begin{tabular}{|l|c|c|c|c|}
\hline & Anzahl & $\begin{array}{c}\text { WR (Mittelwert, } \\
\text { Standardabweichung) in \% }\end{array}$ & $\begin{array}{c}\text { WR min. Wert } \\
\text { in \% }\end{array}$ & $\begin{array}{c}\text { WR max. Wert } \\
\text { in \% }\end{array}$ \\
\hline \hline Kontrollgruppe & 159 & $63,5 \pm 12,1$ & 31,3 & 85,9 \\
\hline LKG unilateral rechts & 23 & $48,2 \pm 14,6$ & 29,3 & 78,8 \\
\hline LKG unilateral links & 32 & $49,9 \pm 17,1$ & 9,1 & 73,7 \\
\hline LKG bilateral & 17 & $44,6 \pm 17,4$ & 11,1 & 67,7 \\
\hline
\end{tabular}

\subsection{Tabelle 3: Vergleich zwischen weiblichen und männlichen Probanden}

\begin{tabular}{|l|c|c|c|}
\hline & Anzahl & $\begin{array}{r}\text { WR (Mittelwert, } \\
\text { Standardabweichung) in \% }\end{array}$ & $\begin{array}{c}\text { Alter (Mittelwert, } \\
\text { Standardabweichung) }\end{array}$ \\
\hline \hline Kontrollgruppe & & & $9,1 \pm 2,9$ \\
\hline \hline weiblich & 75 & $63,2 \pm 13,3$ & $9,1 \pm 3,0$ \\
\hline männlich & 84 & $63,9 \pm 11,1$ & $7,7 \pm 2,3$ \\
\hline LKG unilateral rechts & & & $9,5 \pm 2,7$ \\
\hline \hline weiblich & 9 & $46,0 \pm 14,3$ & $9,2 \pm 3,5$ \\
\hline männlich & 14 & $49,6 \pm 15,2$ & $8,3 \pm 3,2$ \\
\hline LKG unilateral links & & & \\
\hline \hline weiblich & 14 & $56,0 \pm 16,6$ & $8,1 \pm 2,1$ \\
\hline männlich & 18 & $45,3 \pm 16,5$ & $9,1 \pm 3,6$ \\
\hline LKG bilateral & & & $32,8 \pm 18,9$ \\
\hline \hline weiblich & 6 & $51,0 \pm 13,3$ & \\
\hline männlich & 11 & & \\
\hline
\end{tabular}




\section{Abbildungen}

\subsection{Abbildung 1}

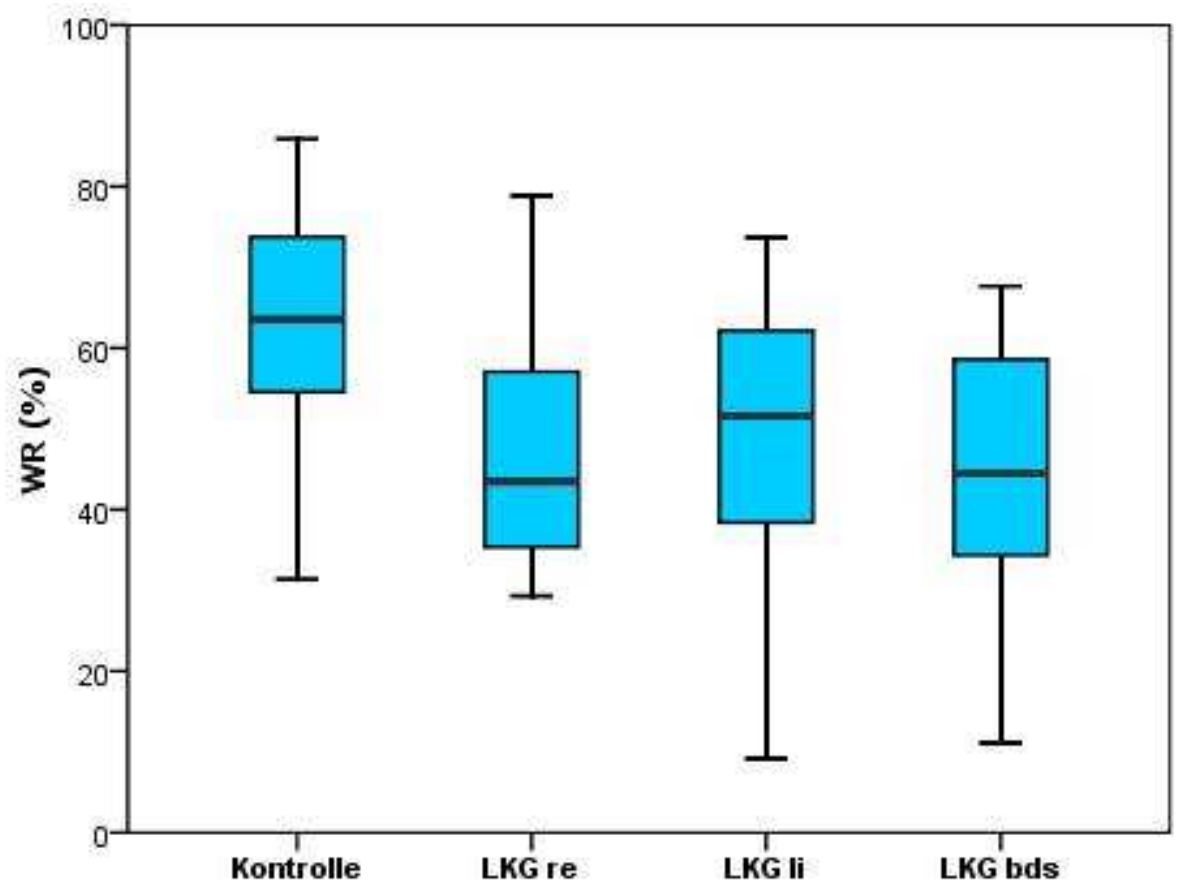

Boxplot der Worterkennungsrate (WR) der Kontrollgruppe (159 Kinder) und bei Kindern mit Lippen-Kiefer-Gaumen-Spaltfehlbildungen (LKG rechts 23 Kinder, LKG links 32 Kinder, LKG beidseits 17 Kinder). Der Mittelwert ist in jeder Box durch einen Querstrich angegeben. Die Box beinhaltet die Kinder innerhalb der mittleren Quartile. 


\subsection{Abbildung 2}

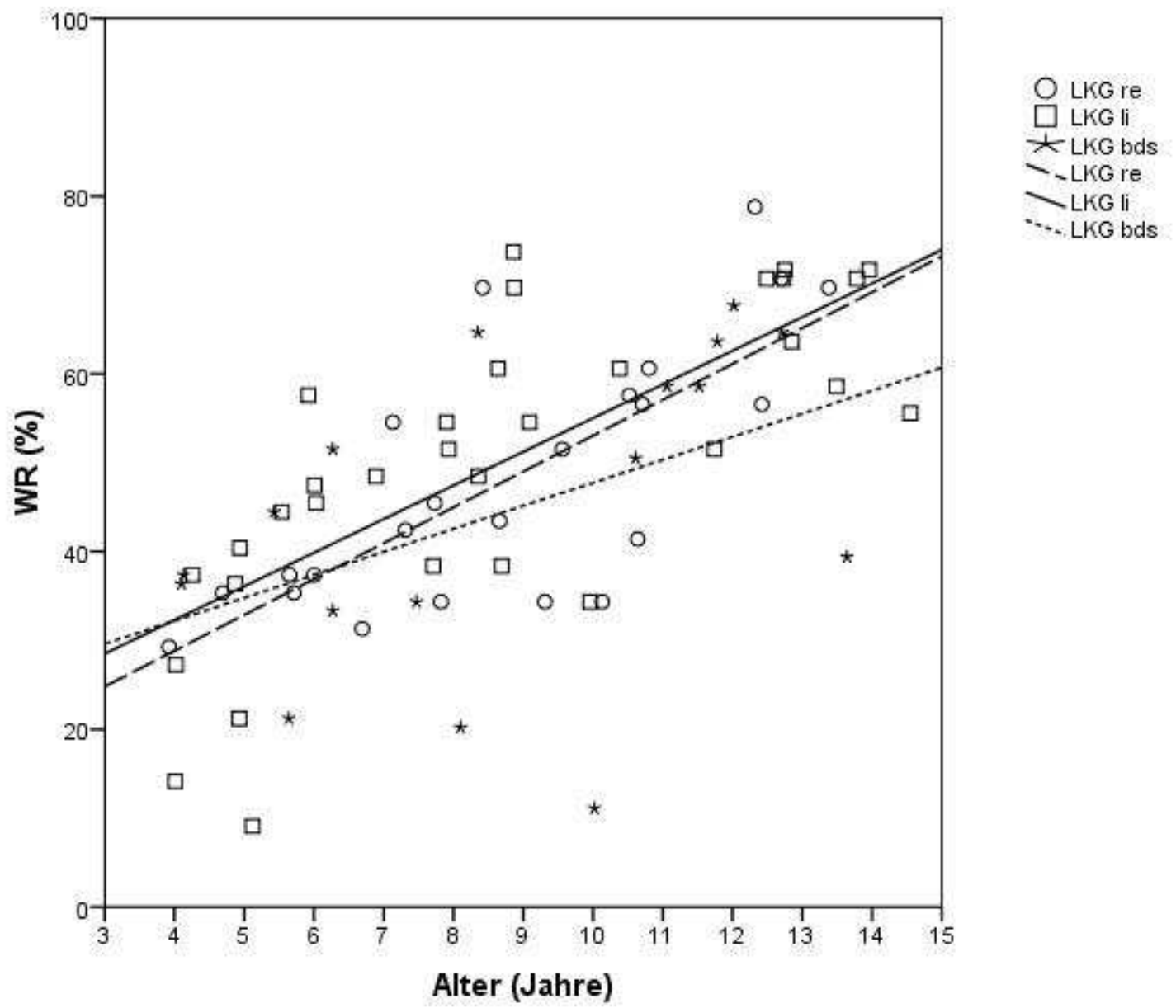

Worterkennungsrate (WR) in Bezug zum Alter der Kinder mit Lippen-KieferGaumenspaltfehlbildung (LKG) rechts, LKG links und LKG beidseits, zudem die jeweils zugehörige Regressionsgerade. 


\section{Literaturverzeichnis}

1. Brunner M, Stellzig-Eisenhauer A, Pröschel U, Verres R, Komposch G. The effect of nasopharyngoscopic biofeedback in patients with cleft palate and velopharyngeal dysfunction. Cleft Palate Craniofac J. 2005 Nov;42(6):649-57.

2. Chapman K, Graham KT, Gooch J, Visconti C. Conversational Skills of Preschool and School-Age Children with Cleft Lip and Palate. Cleft Palate Craniofac J Nov 1998;35(6):503-516

3. Dalston RM, Warren DW, Dalston ET. A preliminary study of nasal airway patency and its potential effect on speech performance. Cleft Palate Craniofac $\mathrm{J}$ 1992;29(4):330-335

4. Fox A.V. PLAKSS - Psycholinguistische Analyse kindlicher Sprechstörungen. Frankfurt am Main: Swets \& Zeitlinger, 2002

5. Frederickson M, Chapman K, Hardin-Jones M. Conversational Skills of Children with Cleft Lip and Palate: A Replication and Extension. Cleft Palate Craniofac J March 2006;43(2):179-188

6. Harding A, Grunwell P. Active versus passive cleft-type speech characteristics. Int J Lang Commun Disord 1998;33(3):329-352

7. Harding A, Grunwell P. Characteristics of cleft palate speech. Eur J Disord Commun 1996;31(4):331-57

8. Karling J, Larson O, Leanderson R, Henningsson G. Speech in Unilateral and Bilateral Cleft Palate Patients from Stockholm. Cleft Palate Craniofac J 1993;30(1):73-77

9. Keuning $\mathrm{KH}$, Wieneke $\mathrm{GH}$, Dejonckere $\mathrm{PH}$. The Intrajudge Reliability of the Perceptual Rating of Cleft Palate Speech Before and After Pharyngeal Flap Surgery: The Effect of Judges and Speech Samples. Cleft Palate Craniofac J 1999;36(4):328-33.

10. Leeper H, Pannbacker M, Roginski J. Oral language characteristics of adult cleftpalate speakers compared on the basis of cleft type and sex. J Commun Disord 1980;13:133-146

11. Lohmander A, Friede H, Elander A, Persson C, Lilja J. Speech development in patients with unilateral cleft lip and palate treated with different delays in closure of the hard palate after early velar repair: a longitudinal perspective. Scand J Plast Reconstr Surg Hand Surg 2006;40(5):267-274

12. Lohmander A, Olsson M. Methodology for perceptual assessment of speech in patients with cleft palate: a critical review of the literature. Cleft Palat Craniofacial $\mathrm{J}$ 2004;41(1):64-70 
13. Lohmander A, Söderpalm E, Friede H, Lilja J. A longitudinal study of speech in 15 children with cleft lip and palate treated by late repair of the hard palate. Scand $\mathrm{J}$ Plast Reconstr Surg Hand Surg Mar 1995;29(1):21-31

14. Maier A, Haderlein T, Eysholdt U, Rosanwoski F, Batliner A, Schuster M, Nöth E. PEAKS - a system for the automatic evaluation of voice and speech disorders. Speech Communication 2009; to appear

15. Maier A, Hönig F, Bocklet T, Stelzle F, Nkenke E, Nöth E, Schuster M. Automatic detection of articulation disorders in children with cleft lip and palate. JASA 2009, to appear

16. Maier A, Nöth E, Batliner A, Nkenke E, Schuster M. Fully Automatic Assessment of Speech of Children with Cleft Lip and Palate. Informatica 2006;30:477-482

17. Middag C, Van Nuffelen G, Martens J-P, de Bodt M. Objective intelligibility assessment of pathologic speakers. In Proc. International Conference on Spoken Language Processing, Brisbane, Australia, September 2008; 1745-1748

18. Nakajima T, Mitsudome A, Yosikawa A. Postoperative speech development based on cleft types in children with cleft palate. Pediatrics Int 2001;43:666-672

19. Paal S, Reulbach U, Strobel-Schwarthoff K, Nkenke E, Schuster M. Bewertung von Sprechauffälligkeiten bei Kindern mit LKG-Spaltfehlbildungn. J Orofac Orthop 2005;66:270-278

20. Rosanowski F, Eysholdt U. Phoniatric aspects in cleft lip patients. Facial Plast Surg 2002;18(3):197-203

21. Schönweiler R, Schönweiler B, Schmelzeisen R, Ptok M. Sprach- und Sprechleistungen bei 417 Kindern mit Spaltbildungen. Fortschr. Kieferorthop. 1995;(1):1-6

22. Schuster M, Haderlein T, Nöth E, Lohscheller J, Eysholdt U, Rosanowski F. Intelligibility of Laryngectomees' Substitute Speech: Automatic Speech Recognition and Subjective Rating. Eur Arch Otorhinolaryngol 2006;263(2):188193

23. Schuster M, Maier A, Haderlein T, Nkenke E, Wohlleben U, Rosanowski F, Eysholdt U, Nöth E. Evaluation of Speech Intelligibility for Children with Cleft Lip and Palate by Means of Speech Recognizer Technique. Int J Pediatr Otorhinolaryngol 2006;70:1741-1747

24. Sell D, Grunwell P, Mildinhall S, Murphy T, Cornish TA, Bearn D, Shaw WC, Murray JJ, Williams AC, Sandy JR. Cleft lip and palate care in the United Kingdom—the Clinical Standards Advisory Group (CSAG) Study. Part 3: speech outcomes. Cleft Palate Craniofac J Jan 2001;38(1):30-37

25. Spriestersbach DC, Moll K, Morris H. Subject Classification and Articulation of 
Speakers with Cleft Palates. J of Speech and Hearing Research 1961;4(4):362372

26. Timmons MJ, Wyatt RA, Murphy T. Speech after repair of isolated cleft palate and cleft lip and palate. Br J Plast Surg 2001;54:377-384

27. Tolarova M, Cervenka J. Classification and birth prevalence of orofacial clefts. Am J Med Genet 1998;75(2):126-137

28. Van Lierde KM, De Bodt M, Baetens I, Schrauwen V, Van Cauwenberge P. Outcome of Treatment regarding Articulation, Resonance and Voice in Flemish Adults with Unilateral and Bilateral Cleft Palate. Folia Phoniatr Logop 2003;55:80$90 \mathrm{~K}$

29. Van Lierde KM, De Bodt M, Van Borsel J, Wuyts FL, Van Cauwenberge P. Effect of cleft type on overall speech intelligibility and resonance. Folia Phoniatr Logop 2002;54(3):158-168.

30. Van Nuffelen G, Middag C, De Bodt M, Martens JP. Speech technology-based assessment of phoneme intelligibility in dysarthria. Int $\mathrm{J}$ Lang Commun Disord 2008;30:1-15

31. Vogt B, Maier A, Batliner A, Nöth E, Nkenke E, Eysholdt U, Schuster M. Numerische Quatifizierung der Verständlichkeit von Schulkindern mit isolierter und kombinierter Gaumenspalte. HNO 2007;55(11):891- 898

32. Whitehill TL, Chau $\mathrm{CH}$. Single-word intelligibility in speakers with repaired cleft palate. Clin Linguist Phon 2004;18(4-5):341-355

33. Wohlleben U. Die Verständlichkeitsentwicklung von Kindern mit Lippen-KieferGaumen-Segel-Spalten. Idstein: Schulz-Kirchner, 2004

34. Wyatt R, Sell D, Russell J, Harding A, Harland K, Albery E. Cleft palate speech dissected: a review of current knowledge and analysis. Br J Plast Surg 1996;49(3):143-149 


\section{Danksagung}

Diese Arbeit wurde von der Deutschen Forschungsgemeinschaft (SCHU 2320/1-1) unterstützt.

Bedanken möchte ich mich bei Herrn Prof. Dr. Dr. U. Eysholdt für die Möglichkeit diese Arbeit in der Phoniatrischen und Pädaudiologischen Abteilung der HNO-Klinik durchführen zu können.

Mein besonderer Dank gilt Frau PD Dr. Maria Schuster für Ihre wertvolle Unterstützung und die außerordentlich gute Betreuung während der gesamten Zeit.

Fern ab vom wissenschaftlichen Bereich möchte ich meinen lieben Eltern danken, die immer ein offenes Ohr für mich hatten und mir in jeder erdenklichen Art und Weise stets zur Seite gestanden haben. 


\section{Lebenslauf}

\section{Persönliche Daten}

Name

Franziska Dames

Geburtsdatum

29. März 1984

Geburtsort

Meerane

Familienstand

ledig

Eltern

Hannelore und Hans-Jürgen Dames

Geschwister

Doreen Dames

\section{Aktuelle Beschäftigung}

seit 02/2009

Vorbereitungsassistentin in der Zahnarztpraxis Dr.

Michaela Prinzessin Wolkonsky, München

\section{Studium}

$04 / 2003-01 / 2009$

Studium der Zahnmedizin an der Friedrich-Alexander-

Universität Erlangen/Nürnberg

Studienabschluss: Staatsexamen

02.03.2004

Naturwissenschaftliche Vorprüfung

28.03.2006

Zahnärztliche Vorprüfung

17.12.2008

Zahnärztliche Prüfung

\section{Schulbildung}

10/1994 - 07/2002

Georgius-Agricola-Gymnasium, Glauchau Abschluss: Allgemeine Hochschulreife

09/1990 - 09/1994

Erich-Weinert-Grundschule, Glauchau

Bisherige Beschäftigung

08/2002 - 03/2003

berufliche Orientierungsphase, während dieser Zeit dauerhafte Nebentätigkeit im Einzelhandel (Kaufland Dienstleistung $\mathrm{GmbH} \&$ Co. $\mathrm{KG}$ ) 